\title{
High-field study of $\mathrm{UCo}_{2} \mathrm{Si}_{2}:$ magnetostriction at metamagnetic transition and influence of Fe substitution
}

\author{
A.V. Andreev ${ }^{\mathrm{a}, 1}$, Y. Skourski ${ }^{\mathrm{b}}$, D.I. Gorbunov ${ }^{\mathrm{a}, \mathrm{b}}$, K. Prokeš $^{\mathrm{c}}$ \\ ${ }^{a}$ Institute of Physics, Academy of Sciences, Na Slovance 2, 18221 Prague, Czech Republic \\ ${ }^{b}$ Hochfeld-Magnetlabor Dresden (HLD-EMFL), Helmholtz-Zentrum, Dresden-Rossendorf, \\ 01328 Dresden, Germany \\ ${ }^{c}$ Helmholtz-Zentrum Berlin fur Materialien und Energie, Hahn-Meitner Platz 1, 14109 \\ Berlin, Germany
}

\begin{abstract}
UCO}_{2} \mathrm{Si}_{2}$ (tetragonal crystal structure) is antiferromagnet below $T_{\mathrm{N}}=83 \mathrm{~K}$ with ferromagnetic basal-plane layers of $U$ magnetic moments oriented parallel to the $c$ axis. The layers are coupled in +-+- sequence along this axis. In fields of $45 \mathrm{~T}$ applied along the $c$ axis, $\mathrm{UCo}_{2} \mathrm{Si}_{2}$ exhibits very sharp metamagnetic transition to ++- uncompensated antiferromagnetic state. The transition is accompanied by pronounced magnetostriction effects. The crystal expands along the $c$ axis by $1 * 10^{-4}$ and shrinks in the basal plane by $0.5 * 10^{-4}$ (at $1.5 \mathrm{~K}$ ) resulting in negligible volume effect. Between $20 \mathrm{~K}$ and $40 \mathrm{~K}$ the transition changes from the first- to the second-order type. The Fe doping in $\mathrm{UCo}_{2} \mathrm{Si}_{2}$ reduces $T_{\mathrm{N}}$ from $83 \mathrm{~K}$ to $80 \mathrm{~K}$ at $x=$ 0.2 in $\mathrm{U}\left(\mathrm{Co}_{1-x} \mathrm{Fe}_{x}\right)_{2} \mathrm{Si}_{2}$. Metamagnetic transition shifts to higher fields (from $45 \mathrm{~T}$ at $x=0$ to $56 \mathrm{~T}$ for $x=0.2$ ). Magnetization jump over the transition remains practically the same which is in agreement with uranium magnetic moment determined by neutron diffraction on crystal with $x=0.1$ as $1.29 \mu_{\mathrm{B}}$, i.e. only slightly lower than that in $\mathrm{UCo}_{2} \mathrm{Si}_{2}$.
\end{abstract}

Key words: Uranium intermetallics; Antiferromagnetism; Magnetostriction; High magnetic fields; Field-induced transitions;

${ }^{1}$ Corresponding author, e-mail: andreev@fzu.cz 


\section{Introduction}

$\mathrm{UCo}_{2} \mathrm{Si}_{2}$ belongs to a wide class of uranium ternary compounds $\mathrm{UT}_{2} \mathrm{X}_{2}$ ( $\mathrm{T}$ is a late $3 \mathrm{~d}$, $4 \mathrm{~d}$, or $5 \mathrm{~d}$-electron metal and $\mathrm{X}$ is a p-electron element $\mathrm{Si}$ or $\mathrm{Ge}$ ) whose magnetic and other electronic properties were studied systematically. These materials exhibit a large variety of magnetic states: antiferromagnets (e.g., $\left.\mathrm{UCr}_{2} \mathrm{Si}_{2}\right)$, uncompensated antiferromagnets $\left(\mathrm{UNi}_{2} \mathrm{Si}_{2}\right)$, ferromagnets $\left(\mathrm{UCu}_{2} \mathrm{Si}_{2}\right)$, Pauli paramagnets $\left(\mathrm{UFe}_{2} \mathrm{Si}_{2}\right)$ and one of the most intriguing cases, $\mathrm{URu}_{2} \mathrm{Si}_{2}$, where heavy-fermion superconductivity combines with so-called hidden order [1-3]. $\mathrm{UCo}_{2} \mathrm{Si}_{2}$ crystallizes in the body-centered variant of tetragonal crystal structure characteristic for $\mathrm{UT}_{2} \mathrm{X}_{2}$, the $\mathrm{ThCr}_{2} \mathrm{Si}_{2}$ type (space group $\mathrm{I} 4 / \mathrm{mmn}$ ). The structure is formed by $\mathrm{U}$, Co, and $\mathrm{Si}$ basal-plane atomic layers stacked along the $c$ axis. $\mathrm{UCo}_{2} \mathrm{Si}_{2}$ is antiferromagnet (AF) below the Néel temperature $T_{\mathrm{N}}=83 \mathrm{~K}$. The magnetic structure obtained from powder neutron diffraction analysis consists of ferromagnetic basal-plane layers of $U$ magnetic moments $M_{U}$ with $1.42 \mu_{\mathrm{B}}$ (at $4.2 \mathrm{~K}$ ) oriented parallel to the $c$ axis that are coupled in an alternating +-+sequence (AF type-I structure) along this axis [4]. The magnetic moments are carried only by the $U$ atoms. This simple magnetic structure exists over the whole temperature interval below $T_{\mathrm{N}}$ without any order-order transition in zero field.

Since a large or even huge magnetic anisotropy is usually observed in uranium magnetically ordered materials, the quantitative data on magnetism of uranium compounds can be collected only on single-crystalline samples. For this reason, measurements of magnetization, electrical resistivity and specific heat were performed on single crystalline samples of $\mathrm{UCo}_{2} \mathrm{Si}_{2}$ [5]. Magnetization and magnetoacoustics were measured in pulsed magnetic fields up to 60-65 $\mathrm{T}$ [6]. In magnetic fields applied along the $c$ axis, $\mathrm{UCo}_{2} \mathrm{Si}_{2}$ undergoes a metamagnetic transition at critical field $\mu_{0} H_{\mathrm{cr}}=45 \mathrm{~T}$ at $1.5 \mathrm{~K}$ (Fig. 1) whereas in fields applied in the basal plane it demonstrates linear magnetization curve with temperatureindependent magnetic susceptibility confirming very large magnetic anisotropy of the compound. The metamagnetic transition is extremely sharp and exhibits a small but nonnegligible hysteresis $(0.2 \mathrm{~T})$ confirming its first order. With increasing temperature, it becomes broader and vanishes at $T_{\mathrm{N}}$. The magnetization gain $\Delta M$ upon the transition, $0.5 \mu_{\mathrm{B}}$ (at $1.5 \mathrm{~K}$ ), corresponds roughly to $1 / 3$ of $U$ magnetic moment found by neutron diffraction. Therefore, the state above the metamagnetic transition is uncompensated antiferromagnetic (UAF) with a ++- arrangement of the magnetic moments. This magnetic structure is known as the ground state in $\mathrm{UNi}_{2} \mathrm{Si}_{2}$, compound isostructural with $\mathrm{UCo}_{2} \mathrm{Si}_{2}$.

Ultrasound measurements confirmed occurrence of this transition which is accompanied 
by very large and sharp anomalies both in changes of sound velocity $\Delta v / v$ and sound attenuation $\Delta a$ [6]. The anomalies have a non-monotonous temperature evolution with maximum effects at $30 \mathrm{~K}$, where $\Delta v / v$ and $\Delta a$ reach very large values of $-4.5 \%$ and 120 $\mathrm{dB} / \mathrm{cm}$, respectively. Whereas $\Delta a$ exhibits only a very sharp peak at the transition, $\Delta v / v$ has a more complicated behavior.

In the present work we studied another magnetoelastic effect, magnetostriction. We performed measurements of both longitudinal and transverse magnetostriction in pulsed fields up to $60 \mathrm{~T}$ applied along the $c$ axis. We also studied the influence of the Fe doping on the high-field magnetization of $\mathrm{UCo}_{2} \mathrm{Si}_{2}$. Magnetic structure of a representative compound, $\mathrm{UCo}_{1.8} \mathrm{Fe}_{0.2} \mathrm{Si}_{2}$, was investigated by neutron diffraction on a single crystal.

\section{Experimental}

Single crystals $\mathrm{U}\left(\mathrm{Co}_{1-x} \mathrm{Fe}_{x}\right)_{2} \mathrm{Si}_{2}$ with $x=0,0.05,0.1$ and 0.2 were grown by a modified Czochralski method in a tri-arc furnace on a copper water-cooled bottom from mixtures of the pure elements, 99.9\% U, 99.99\% Fe and Co, and 99.999\% Si. The pulling of the crystals was performed under argon protecting atmosphere at a speed of $10 \mathrm{~mm} / \mathrm{h}$ with a tungsten rod as a seed. The resulting single crystals were cylinder-shaped with a height of $20 \mathrm{~mm}$ and a diameter up to $5 \mathrm{~mm}$. Standard x-ray powder diffraction analysis confirmed a single-phase state in the tetragonal crystal structure of the $\mathrm{ThCr}_{2} \mathrm{Si}_{2}$ type. Lattice parameters change by less than $0.2 \%$ ( $a$ increases, $c$ decreases) up to $x=0.2$. Back-scattered Laue diffraction was used to check the single-crystalline state and to orient the crystals for measurements. As an example, Fig. 2 shows Laue patterns for $a$ and $c$ axes of one of the crystals, $\mathrm{UCo}_{1.8} \mathrm{Fe}_{0.2} \mathrm{Si}_{2}$, which confirm high quality of the crystal.

Temperature and field dependences of magnetization in static magnetic fields up to 14 T were measured using PPMS-14 cryomagnetic installation (Quantum Design). High-field magnetization was measured in pulsed magnetic fields up to $60 \mathrm{~T}$ (pulse duration $20 \mathrm{~ms}$ ) by the induction method using a coaxial pick-up coil system. The high-field magnetometer is described in detail in [7]. Absolute values of the magnetization were calibrated using data from measurements in static magnetic field.

The magnetostriction was measured using an optical fiber strain gauge bonded to the surface of the sample with cyanoacrylate epoxy. The strain gauge consists of a $1 \mathrm{~mm}$ long fiber Bragg grating written into the core of a standard telecommunication fiber, with a peak reflectivity at $1550 \mathrm{~nm}$. The strain-dependent shift of the peak in the reflectivity is detected 
using a high-resolution grating spectrometer. The method is described in Ref. [8]

Neutron diffraction experiments on the $\mathrm{UCo}_{1.8} \mathrm{Fe}_{0.2} \mathrm{Si}_{2}$ single crystal were carried out on the normal-beam diffractometer E4 installed at the BER II reactor of the HelmholtzZentrum Berlin. This instrument uses a PG(002)-monochromator selecting the neutron wavelength $\lambda=2.41 \AA$. The data can be collected with a two-dimensional position sensitive ${ }^{3}$ He-detector, $200 \times 200 \mathrm{~mm}(256 \times 256$ pixels $)$. Normally, only reflections close to the scattering plane, that is perpendicular to the rotational axis of the diffractometer are collected. In order to refine the magnetic structure of $\mathrm{U}\left(\mathrm{Co}_{0.9} \mathrm{Fe}_{0.1}\right)_{2} \mathrm{Si}_{2}$ we have collected all reachable reflections within the $(h 0 l)$ plane at $2 \mathrm{~K}$ produced by a standard orange-type cryostat using a single crystal with approximate dimensions $2 \times 1 \times 1 \mathrm{~mm}^{3}$. Bragg reflections for the refinement were collected for 20 minutes, each. The temperature dependence of the strongest magnetic reflection was followed at the top of the reflection up to temperature well above the magnetic ordering temperature, for half a minute per temperature point. Both, the crystal and magnetic structure refinements were carried out with the program Fullprof [9]. The nuclear scattering lengths $b(\mathrm{Si})=4.149 \mathrm{fm}, b(\mathrm{Co})=2.49 \mathrm{fm}, b(\mathrm{Fe})=9.45 \mathrm{fm}$, and $b(\mathrm{U})=8.417 \mathrm{fm}$ were used [10]. A standard $\mathrm{U}^{3+}$ free ion magnetic form factor was used.

\section{Results and discussion}

\subsection{Magnetostriction in $\mathrm{UCo}_{2} \mathrm{Si}_{2}$}

Figure 3 shows linear and volume magnetostriction in the $\mathrm{UCo}_{2} \mathrm{Si}_{2}$ single crystal upon metamagnetic transition at $1.5 \mathrm{~K}$. The magnetization jump is accompanied by well pronounced expansion of the crystal along the $c$ axis by $\lambda_{c}=1 * 10^{-4}$ and shrinkage in the basal plane by $\lambda_{a}=-0.5^{*} 10^{-4}$ thus resulting in a negligible volume effect $\omega=\lambda_{c}+2 \lambda_{a}<1 * 10^{-5}$. Linear strains are rather large, but uranium compounds can exhibit much larger linear magnetostriction reaching so-called giant-magnetostriction values. For example, ferromagnetic compound $\mathrm{UGa}_{2}$ demonstrates an orthorhombic distortion of magnetostrictive origin described by magnetostrictive constant $\lambda^{\gamma, 2}=-4 * 10^{-3}$ at $4.2 \mathrm{~K}$ [11], i.e., more than an order larger than we observed in $\mathrm{UCo}_{2} \mathrm{Si}_{2}$. However, in $\mathrm{UGa}_{2}$ the effect is determined by anisotropic magnetostriction which is very large in the f-electron systems, both in $4 \mathrm{f}-$ and $5 \mathrm{f}$ metal compounds. At the metamagnetic transition in $\mathrm{UCo}_{2} \mathrm{Si}_{2}$ we deal purely with magnetostriction of the exchange origin (so-called isotropic magnetostriction). Since all magnetic moments in $\mathrm{UCo}_{2} \mathrm{Si}_{2}$ below and above the metamagnetic transition are directed along the $c$ axis, anisotropic magnetostriction does not contribute to the observed effect. 
Exchange magnetostriction can be very large when magnetism of the compound is determined by 3d electrons. For example, magnetostriction at metamagnetic transition reaches $4 * 10^{-3}$ in $\mathrm{FeRh}$ [12] or in $\left(\mathrm{Lu}_{0.8} \mathrm{Ce}_{0.2}\right)_{2} \mathrm{Fe}_{17}$ [13]. However, it is much lower in the $4 \mathrm{f}$ or $5 \mathrm{f}$ electron systems. Thermal expansion of $\mathrm{UNi}_{2} \mathrm{Si}_{2}$, compound isostructural with $\mathrm{UCo}_{2} \mathrm{Si}_{2}$, shows anomalies along the $a$ and $c$ axes at $43 \mathrm{~K}$ where the magnetic structure spontaneously changes with cooling from antiferromagnetic +-+- to UAF ++-, i.e., in the same way as in $\mathrm{UCo}_{2} \mathrm{Si}_{2}$ at the metamagnetic transition. $\mathrm{UNi}_{2} \mathrm{Si}_{2}$ shrinks with cooling at $45 \mathrm{~K}$ by $\sim 0.4 * 10^{-4}$ along the $a$ axis and elongates by $\sim 0.3^{*} 10^{-4}$ along the $c$ axis [14]. So, longitudinal and transverse linear magnetostrictions correspond well qualitatively (shrinkage in the basal plane and expansion along the $c$ axis) and quantitatively to field-induced anomalies in $\mathrm{UCo}_{2} \mathrm{Si}_{2}$. Different signs of the linear strains, their magnitudes and negligible volume effect also resemble the behavior of another uranium antiferromagnetic intermetallic, UNiGa, at the metamagnetic transition from the antiferromagnetic to the forced ferromagnetic state which occurs in as low as $1 \mathrm{~T}$ field [15]. Uranium magnetic moment in UNiGa is $1.4 \mu_{\mathrm{B}}$, i.e. practically the same as in $\mathrm{UCo}_{2} \mathrm{Si}_{2}$. It has also huge uniaxial magnetic anisotropy. Magnitude of the linear strain along the $c$ axis reaches $2.2 * 10^{-4}$ in UNiGa, i.e., more than twice larger than in $\mathrm{UCo}_{2} \mathrm{Si}_{2}$. But in $\mathrm{UNiGa}$ the state after the transition is ferromagnetic whereas in $\mathrm{UCo}_{2} \mathrm{Si}_{2}$ it is $\mathrm{UAF}$ with magnetic moment 3 times lower than in forced ferromagnetic state. In final parallel magnetic arrangement $\lambda_{c}$ could be $3 * 10^{-4}$, i.e. not much different from that in UNiGa, especially taking into account different compositions and crystal structures.

Magnetostriction measurements at elevated temperatures (Fig. 4) confirmed that between $20 \mathrm{~K}$ and $40 \mathrm{~K}$ the transition changes its type from the first order to the second order, as was observed also in the magnetization and magnetoacoustic measurements [6]. In contrast to magnetoacoustic characteristics, magnetostrictive strains decrease monotonously with increasing temperature.

\subsection{Influence of $\mathrm{Fe}$ on magnetism of $\mathrm{UCo}_{2} \mathrm{Si}_{2}$}

In addition to a study of magnetostriction at metamagnetic transition in $\mathrm{UCo}_{2} \mathrm{Si}_{2}$, we checked also the influence of the Fe doping on the high-field magnetization of $\mathrm{UCo}_{2} \mathrm{Si}_{2}$. It is known that the terminal compound in the $\mathrm{U}\left(\mathrm{Co}_{1-x} \mathrm{Fe}_{x}\right)_{2} \mathrm{Si}_{2}$ series, $\mathrm{UFe}_{2} \mathrm{Si}_{2}$, is a Pauli paramagnet with temperature-independent susceptibility and without any trace of magnetic ordering [16]. In the isostructural quasiternary system $\mathrm{U}\left(\mathrm{Mn}_{1-x} \mathrm{Fe}_{x}\right)_{2} \mathrm{Si}_{2}$, the strong ferromagnetism observed in $\mathrm{UMn}_{2} \mathrm{Si}_{2}$ is rapidly suppressed with substitutions of $\mathrm{Mn}$ by $\mathrm{Fe}$ and the Curie temperature is approaching zero already around $x=0.4$ [17]. In contrast to such 
behavior, we found that Fe influences magnetism of $\mathrm{UCo}_{2} \mathrm{Si}_{2}$ rather slightly, at least up to $x=$ 0.2 (we stopped at this Fe content due to shift of transition field to unavailable values above $60 \mathrm{~T}$, see below). This difference should be attributed to the magnetic state of $\mathrm{Mn}$ in $\mathrm{UMn}_{2} \mathrm{Si}_{2}$ whereas $\mathrm{Co}$ and $\mathrm{Fe}$ are non-magnetic in $\mathrm{UT}_{2} \mathrm{Si}_{2}$.

Figure 5 shows the temperature dependence of magnetic susceptibility measured along the $c$ axis of $\mathrm{U}\left(\mathrm{Co}_{1-x} \mathrm{Fe}_{x}\right)_{2} \mathrm{Si}_{2}$ single crystals. One can see that the Néel temperature, corresponding to maximum of $\chi(T)$, does not change at $x=0.05$ and 0.1 and decreases to 80 $\mathrm{K}$ at $x=0.2$. In the field applied along the $a$ axis, almost temperature-independent susceptibility observed in $\mathrm{UCo}_{2} \mathrm{Si}_{2}$ does not change with Fe substitution within experimental error. On the other hand, as seen from Fig. 6, the metamagnetic transition shifts from $45 \mathrm{~T}$ at $x$ $=0$ to higher fields (56 $\mathrm{T}$ for $x=0.2$ ). The transition becomes less sharp than in $\mathrm{UCo}_{2} \mathrm{Si}_{2}$ due to some atomic disorder in the Co-Fe sublattice. Presumably for the same reason, hysteresis of the transition increases from $0.2 \mathrm{~T}$ at $x=0$ to $0.7 \mathrm{~T}$ at $x=0.2$. Magnetization jump over the transition remains practically the same, about $0.5 \mu_{\mathrm{B}}$. Temperature evolution of the metamagnetic transition shown in Fig. 6 for the compound with $x=0.2$ is the same as $\mathrm{UCo}_{2} \mathrm{Si}_{2}$ (Fig. 1). The transition changes its type from the first order to the second order between $20 \mathrm{~K}$ and $40 \mathrm{~K}$ and disappears at $T_{\mathrm{N}}$ (Fig. 7).

The $\mathrm{U}\left(\mathrm{Co}_{0.9} \mathrm{Fe}_{0.1}\right)_{2} \mathrm{Si}_{2}$ single crystal was tested by neutron diffraction. A crystalstructure study above $T_{\mathrm{N}}$ confirmed the $\mathrm{ThCr}_{2} \mathrm{Si}_{2}$ type (space group $I 4 / m m n$, No. 139). The U, $\mathrm{Co}(\mathrm{Fe})$ and $\mathrm{Si}$ atoms are at the Wyckoff positions $2 a(0,0,0), 4 d(0,1 / 2,1 / 4)$, and $4 e(0,0, z)$, respectively, where $z$ is determined as 0.379 . The Co and Fe are found to be randomly distributed over the $4 e$ sites. The lattice parameters are $a=388.9 \mathrm{pm}$ and $c=958.3 \mathrm{pm}$ at $2 \mathrm{~K}$. Apart from reflections observed at high temperature, new reflections that violate extinction rules for the $\mathrm{ThCr}_{2} \mathrm{Si}_{2}$ type of structure appear at low temperatures. These appear at positions ( $h 0 l$ ) and (h00) with $h+l=2 n+1$ and $h=2 n+1$, respectively. No intensity is observed at (00l) reflections with $l=2 n+1$. This finding is entirely in agreement with the +-+- sequence (AF type-I structure) observed in $\mathrm{UCo}_{2} \mathrm{Si}_{2}$. A set of magnetic reflections collected at $2 \mathrm{~K}$ leads to $U$ magnetic moment of $1.29(3) \mu_{B}$ which is close to value of $1.42(5) \mu_{B}$ determined for $\mathrm{UCo}_{2} \mathrm{Si}_{2}$ by powder neutron diffraction [4]. Figure 8 shows the temperature dependence of the intensity of the strongest magnetic reflection (100). Value of $T_{\mathrm{N}}=84 \mathrm{~K}$ agrees result obtained from the magnetic susceptibility (83 K).

\section{Conclusions}


$\mathrm{UCo}_{2} \mathrm{Si}_{2}$ (tetragonal crystal structure of the $\mathrm{ThCr}_{2} \mathrm{Si}_{2}$ type) is antiferromagnet below $T_{\mathrm{N}}$ $=83 \mathrm{~K}$. The magnetic structure consists of ferromagnetic basal-plane layers of $\mathrm{U}$ magnetic moments oriented parallel to the $c$ axis. The layers are coupled in +-+- sequence along this axis. In fields of $45 \mathrm{~T}$ applied along the $c$ axis, $\mathrm{UCo}_{2} \mathrm{Si}_{2}$ exhibits very sharp metamagnetic transition to the UAF state with ++- arrangement of the magnetic moments.

In the present work it was found that the magnetization jump is accompanied by well pronounced effects not only in acoustics (sound velocity and attenuation) but also in another magnetoelastic property, magnetostriction. At the transition the $\mathrm{UCo}_{2} \mathrm{Si}_{2}$ crystal expands along the $c$ axis by $\lambda_{c}=1 * 10^{-4}$ and shrinks in the basal plane by $\lambda_{a}=0.5^{*} 10^{-4}$ (at $1.5 \mathrm{~K}$ ) resulting in negligible volume effect $\omega=\lambda_{c}+2 \lambda_{a}<1 * 10^{-5}$. Magnetostriction data confirm that between $20 \mathrm{~K}$ and $40 \mathrm{~K}$ the transition changes from the first-order to the second-order type, as follows also from the magnetization and magnetoacoustic results for $\mathrm{UCo}_{2} \mathrm{Si}_{2}$. Different signs of the linear effects, their magnitudes and low volume effect resemble the behavior of the other uranium antiferromagnetic intermetallics at the metamagnetic transitions.

The Fe doping of $\mathrm{UCo}_{2} \mathrm{Si}_{2}$ reduces $T_{\mathrm{N}}$ from $83 \mathrm{~K}$ only to $80 \mathrm{~K}$ at $x=0.2$ in $\mathrm{U}\left(\mathrm{Co}_{1}\right.$ $\left.{ }_{x} \mathrm{Fe}_{x}\right)_{2} \mathrm{Si}_{2}$. Metamagnetic transition shifts to higher fields (from $45 \mathrm{~T}$ at $x=0$ to $56 \mathrm{~T}$ for $x=$ $0.2)$. Magnetization jump over the transition remains practically the same ( $\left.0.5 \mu_{\mathrm{B}}\right)$. This is in agreement with uranium magnetic moment determined by neutron diffraction on crystal with $x=0.1$ as $1.29 \mu_{\mathrm{B}}$. i.e. only slightly lower than that in $\mathrm{UCo}_{2} \mathrm{Si}_{2}$.

\section{Acknowledgements}

The work was supported by the Materials Growth and Measurement Laboratory (https://mgml.eu) and grant 16-03593S of the Czech Science Foundation. We acknowledge the support of HLD at HZDR, member of the European Magnetic Field Laboratory (EMFL). 


\section{References}

[1] V. Sechovsky, L. Havela, in K.H.J. Buschow (Ed.), Handbook of Magnetic Materials, Vol. 11, Elsevier Science B.V., Amsterdam, 1998, p. 1, and references therein.

[2] A. Szytula, J. Leciejewicz, in K.A. Gschneider, Jr. and L. Eyring (Eds.), Handbook on the Physics and Chemistry of the Rare Earths, Vol. 12, Elsevier Science B.V., Amsterdam, 1989, p. 133, and references therein.

[3] J.A. Mydosh, P.M. Oppeneer, Rev. Mod. Phys. 83 (2011) 1301.

[4] L. Chelmicki, J. Leciejewicz, A. Zygmunt, J. Phys. Chem. Solids 46 (1985) 529.

[5] M. Mihalik, O. Kolomiyets, J.-C. Griveau, A.V. Andreev, V. Sechovský, J. Phys. Soc. Jpn. 76, Suppl. A (2007) 54.

[6] A.V. Andreev, S. Yasin, Y. Skourski, A.A. Zvyagin, S. Zherlitsyn, J. Wosnitza, Phys. Rev. B 87 (2013) 214409.

[7] Y. Skourski, M. D. Kuz’min, K. P. Skokov, A. V. Andreev, J. Wosnitza, Phys. Rev. B 83 (2011) 214420.

[8] R. Daou, F. Weickert, M. Nicklas, F. Steglich, A. Haase, M. Doerr, Rev. Sci. Instrum. 81 (2010) 033909.

[9] S.R. Hall, G.S.D. King, J.M. Stewart, Eds., Xtal3.4 User’s Manual. University of Australia: Lamb, Perth (1995).

[10] V.F. Sears, in: International Tables of Crystallography, vol. C, ed. A.J.C. Wilson (Kluwer, Dordrecht, 1992) p. 383.

[11] A.V. Andreev, K.P. Belov, A.V. Deryagin, Z.A. Kazei, R.Z. Levitin, A. Menovsky, Yu.F. Popov, V.I. Silant’ev, Sov. Phys. JETP 48 (1978) 1187.

[12] R.Z. Levitin, B.K. Ponomarev, Sov. Phys. JETP 23 (1966) 984.

[13] A.V. Andreev, K. Koyama, E.A. Tereshina, J. Prokleška, K. Watanabe, J. Alloys Comp. 477 (2009) 62.

[14] F. Honda, G. Oomi, A.V. Andreev, V. Sechovský, A.A. Menovsky, Physica B 259-261 (1999) 256.

[15] A.V. Andreev, M.I. Bartashevich, T. Goto, J. Alloys Comp. 219 (1995) 267.

[16] A. Szytula, S. Siek, J. Leciejewicz, A. Zygmund, Z. Ban, J. Phys. Chem. Solids 49 (1988) 1113.

[17] W. Bazela, A. Szytula, J. Magn. Magn. Mater. 82 (1989) 151. 


\section{Figure captions}

Fig. 1. Magnetization curves of a $\mathrm{UCo}_{2} \mathrm{Si}_{2}$ single crystal in fields applied along the $c$ axis at $1.5 \mathrm{~K}$ and elevated temperatures. The curve along the $a$ axis at $1.5 \mathrm{~K}$ is also shown.

Fig. 2. Back-scattered Laue patterns for the $a$ and $c$ axes of a $\mathrm{UCo}_{1.8} \mathrm{Fe}_{0.2} \mathrm{Si}_{2}$ single crystal.

Fig. 3. Longitudinal $\left(\lambda_{c}\right)$, transverse $\left(\lambda_{a}\right)$ and volume $(\omega)$ magnetostriction in fields applied along the $c$ axis of a $\mathrm{UCo}_{2} \mathrm{Si}_{2}$ single crystal at $1.5 \mathrm{~K}$.

Fig. 4. Temperature evolution of the longitudinal $\left(\lambda_{c}\right)$ and transverse $\left(\lambda_{a}\right)$ magnetostriction in fields applied along the $c$ axis of a $\mathrm{UCo}_{2} \mathrm{Si}_{2}$ single crystal.

Fig. 5. Temperature dependence of the magnetic susceptibility of $\mathrm{U}\left(\mathrm{Co}_{1-\mathrm{x}} \mathrm{Fe}_{\mathrm{x}}\right)_{2} \mathrm{Si}_{2}$ single crystals in field of $14 \mathrm{~T}$ applied along the $c$ axis.

Fig. 6. Magnetization curves of $\mathrm{U}\left(\mathrm{Co}_{1-\mathrm{x}} \mathrm{Fe}_{\mathrm{x}}\right)_{2} \mathrm{Si}_{2}$ single crystals in fields applied along the $c$ axis at $1.5 \mathrm{~K}$.

Fig. 7. Magnetization curves of a $\mathrm{UCo}_{1.6} \mathrm{Fe}_{0.4} \mathrm{Si}_{2}$ single crystal in fields applied along the $c$ axis at several temperatures.

Fig. 8. The temperature dependence of the intensity of the (100) magnetic Bragg reflection in a $\mathrm{UCo}_{1.8} \mathrm{Fe}_{0.2} \mathrm{Si}_{2}$ single crystal. 


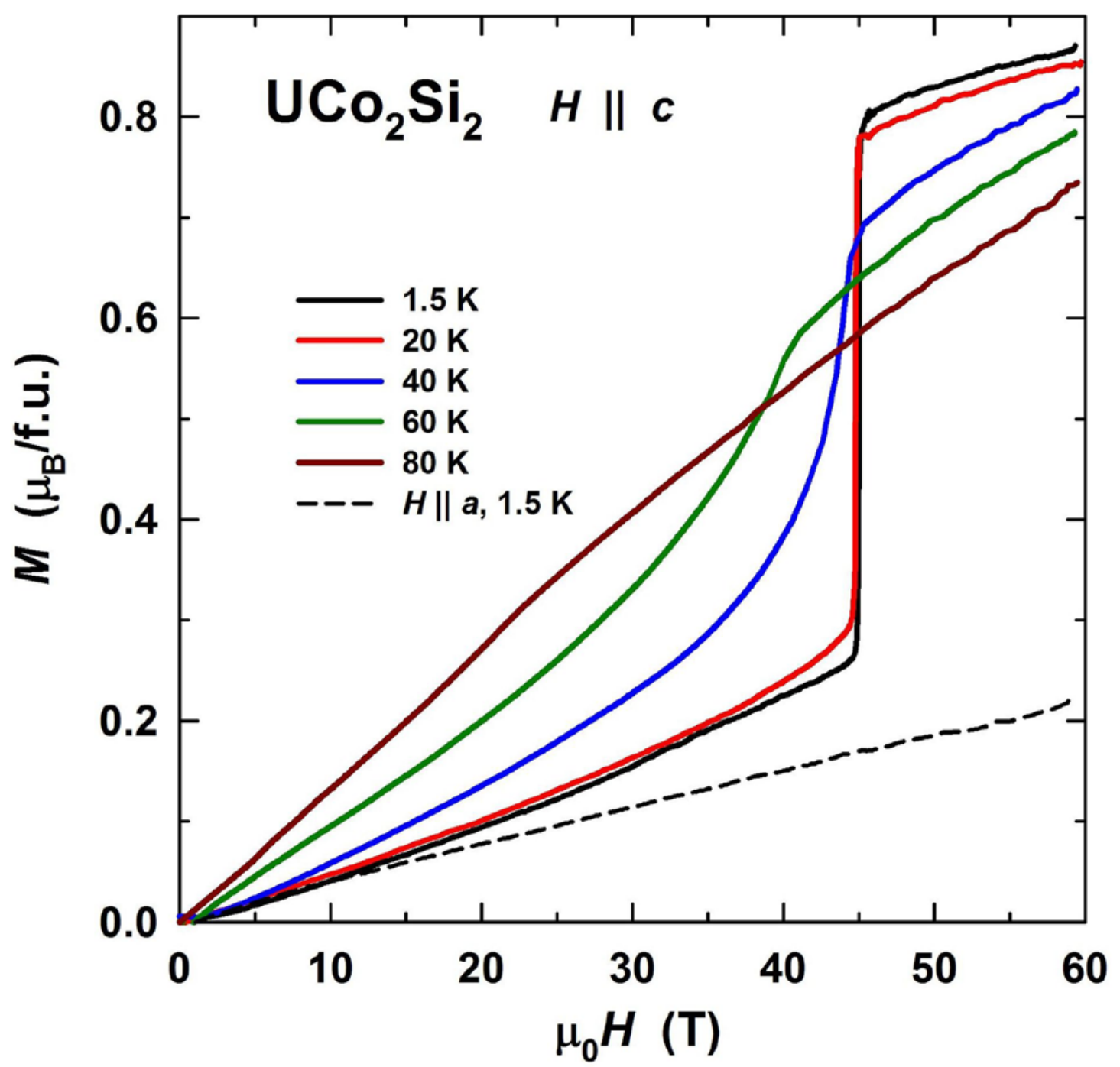

Fig. 1. Magnetization curves of a $\mathrm{UCo}_{2} \mathrm{Si}_{2}$ single crystal in fields applied along the $c$ axis at $1.5 \mathrm{~K}$ and elevated temperatures. The curve along the $a$ axis at $1.5 \mathrm{~K}$ is also shown. 

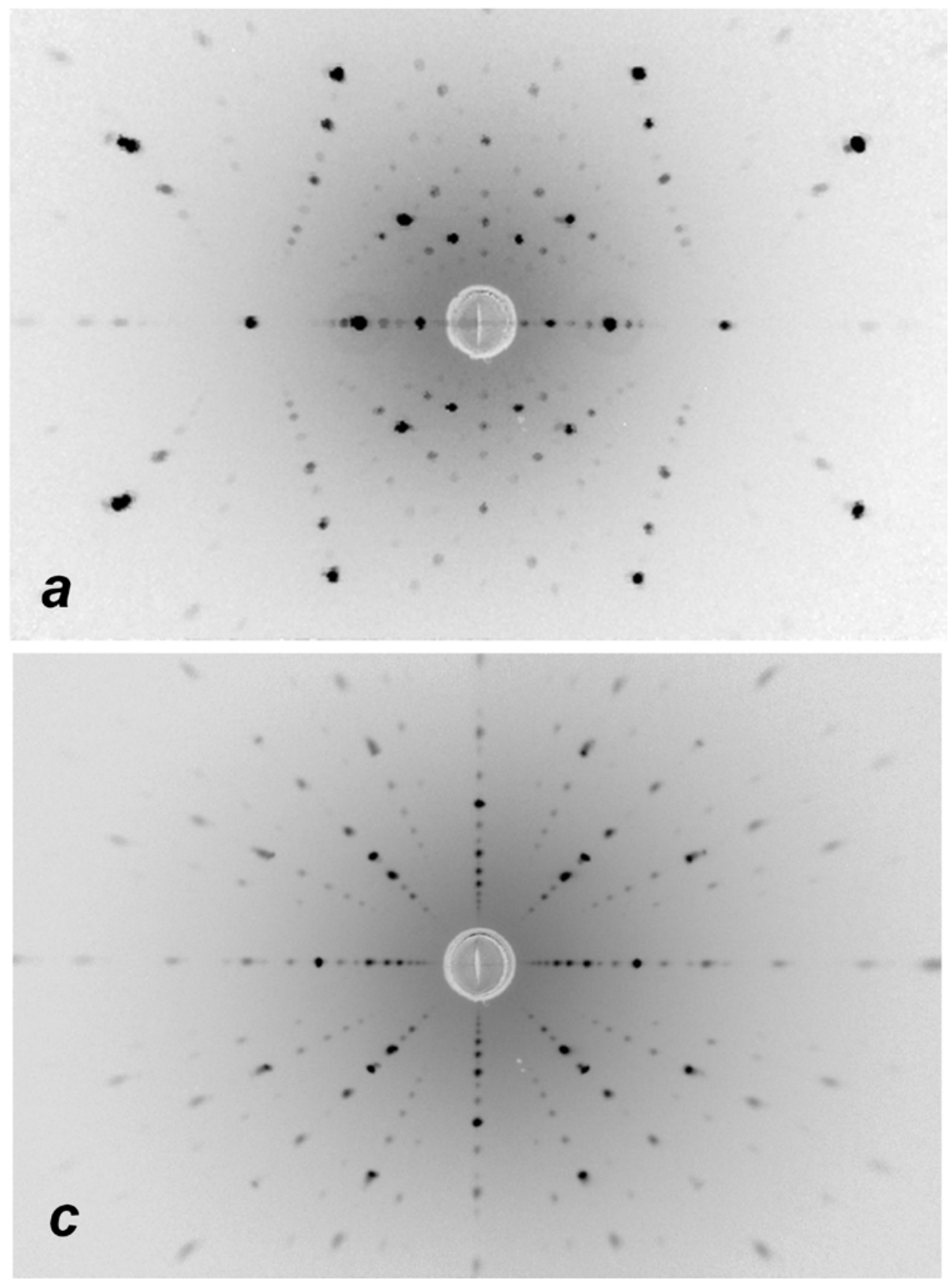

Fig. 2. Back-scattered Laue patterns for the $a$ and $c$ axes of a $\mathrm{UCo}_{1.8} \mathrm{Fe}_{0.2} \mathrm{Si}_{2}$ single crystal. 


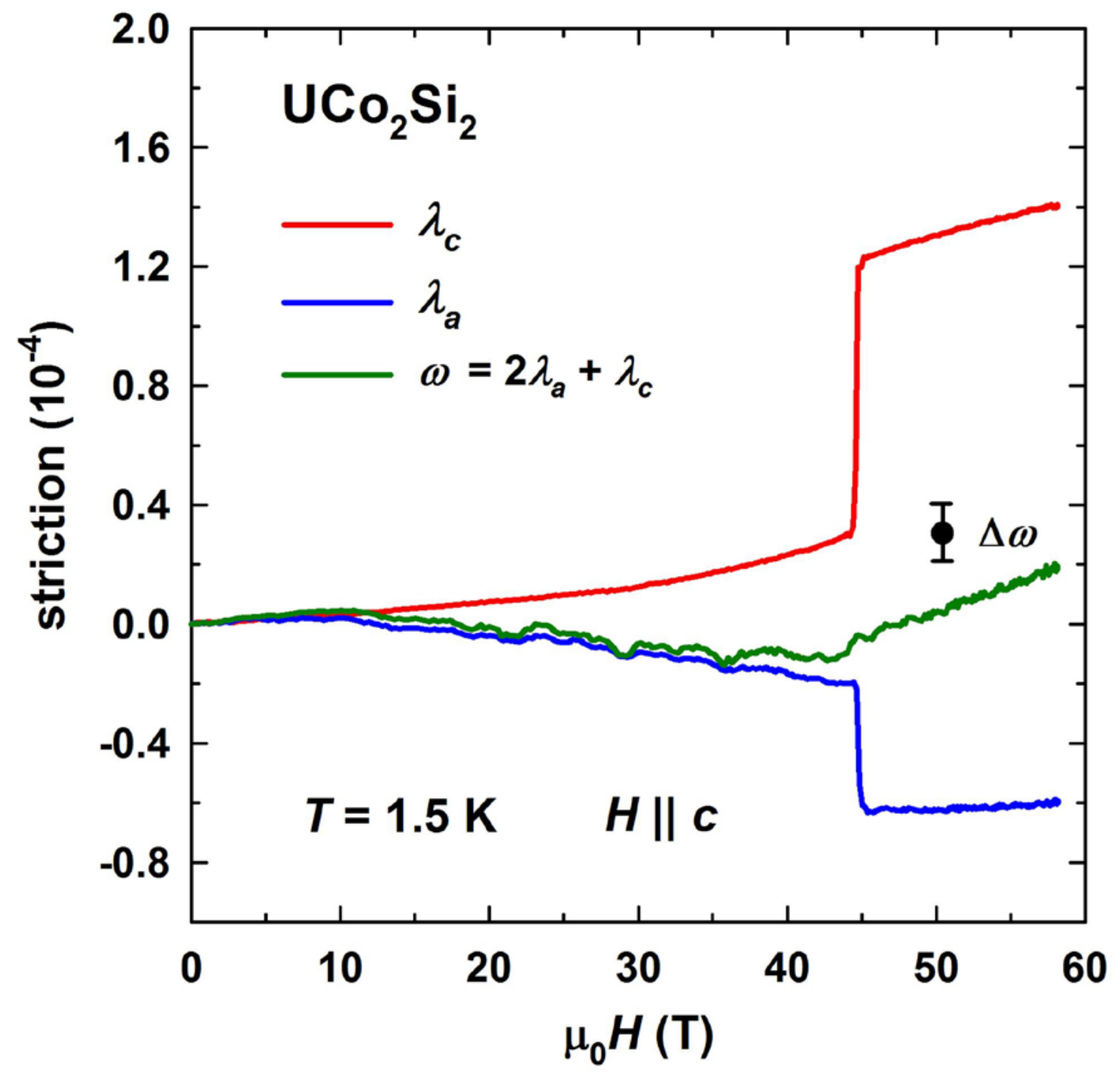

Fig. 3. Longitudinal $\left(\lambda_{c}\right)$, transverse $\left(\lambda_{a}\right)$ and volume $(\omega)$ magnetostriction in fields applied along the $c$ axis of a $\mathrm{UCo}_{2} \mathrm{Si}_{2}$ single crystal at $1.5 \mathrm{~K}$. 


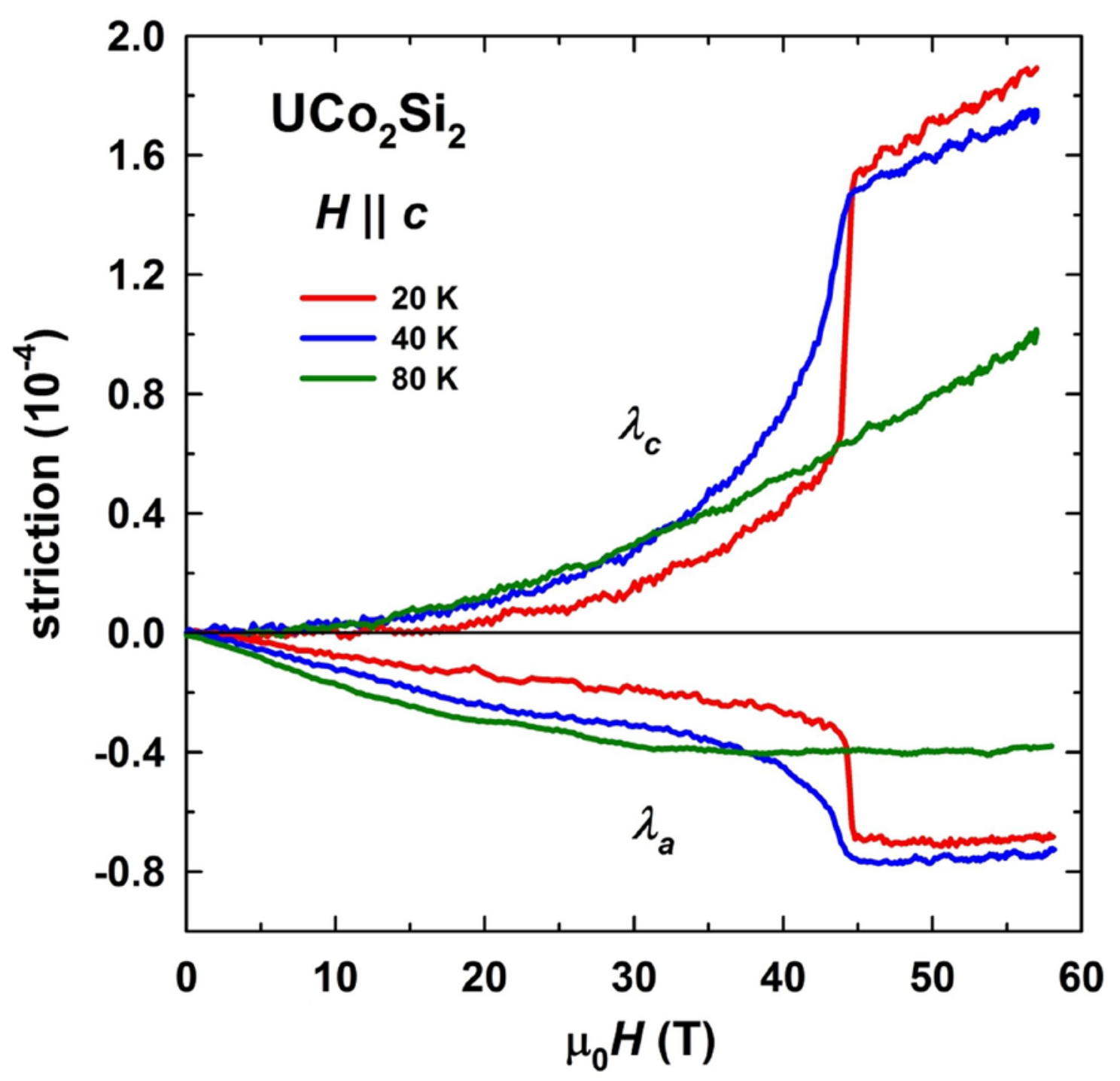

Fig. 4. Temperature evolution of the longitudinal $\left(\lambda_{c}\right)$ and transverse $\left(\lambda_{a}\right)$ magnetostriction in fields applied along the $c$ axis of a $\mathrm{UCo}_{2} \mathrm{Si}_{2}$ single crystal. 


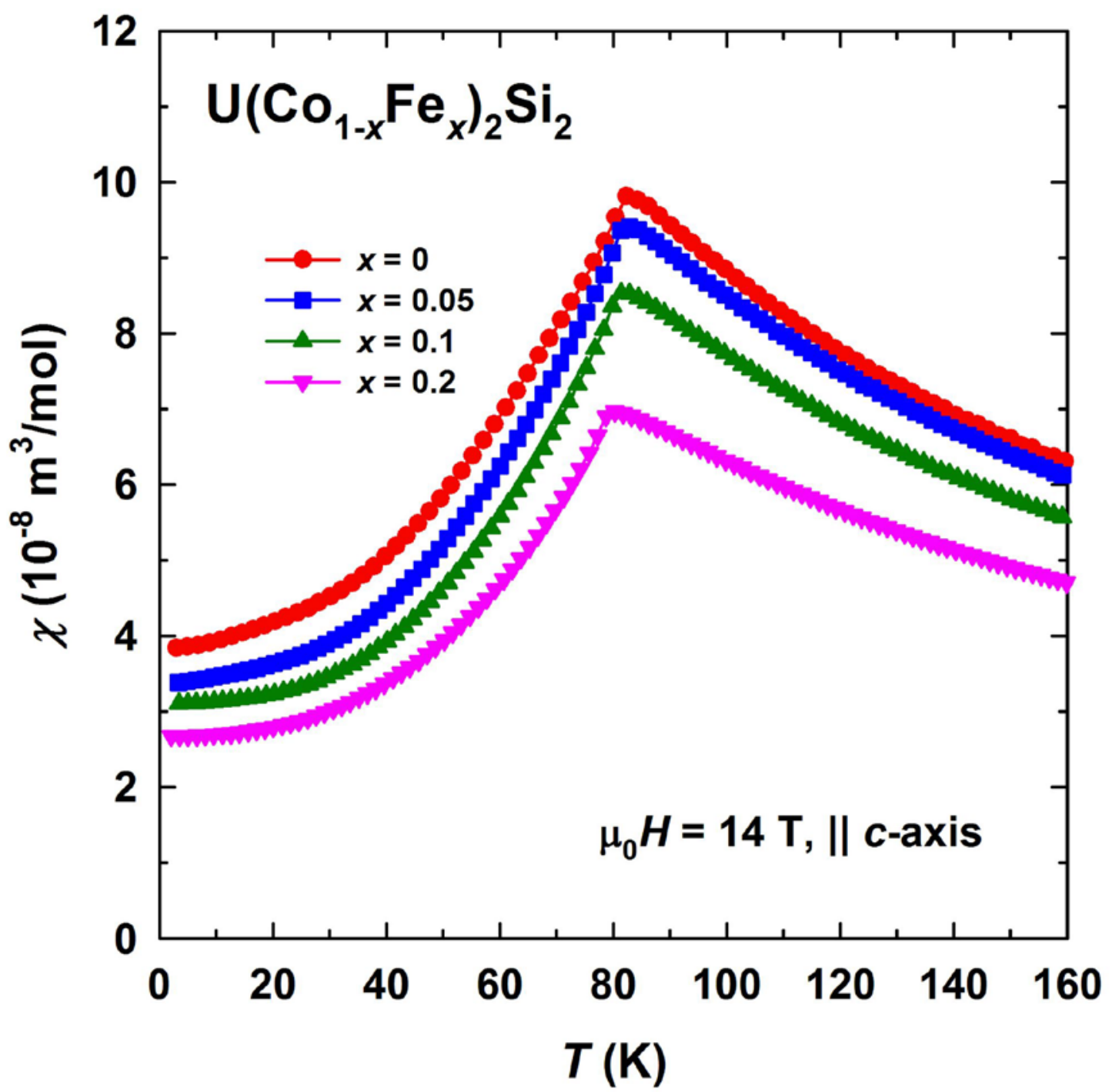

Fig. 5. Temperature dependence of the magnetic susceptibility of $\mathrm{U}\left(\mathrm{Co}_{1-\mathrm{x}} \mathrm{Fe}_{\mathrm{x}}\right)_{2} \mathrm{Si}_{2}$ single crystals in field of $14 \mathrm{~T}$ applied along the $c$ axis. 


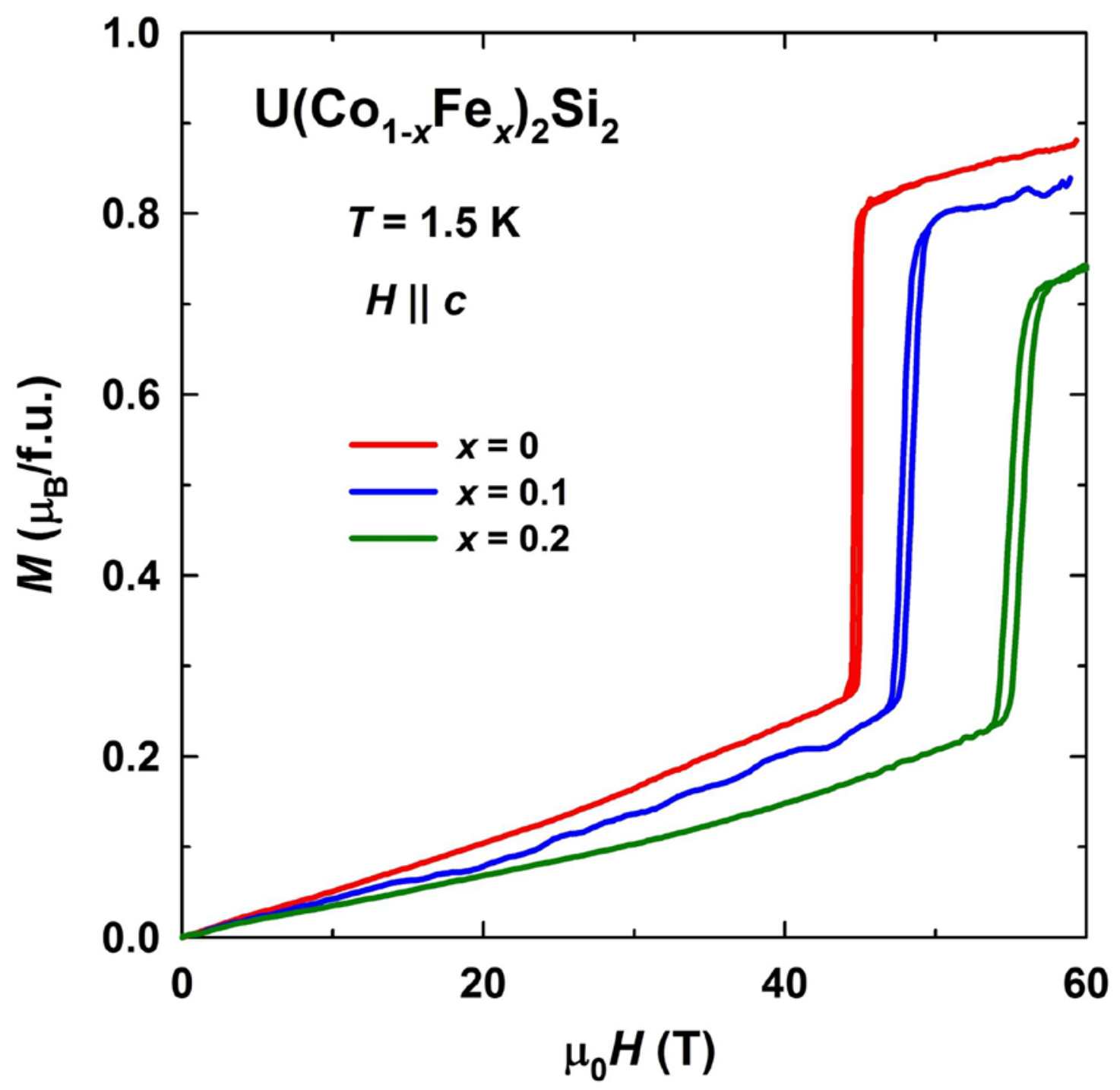

Fig. 6. Magnetization curves of $\mathrm{U}\left(\mathrm{Co}_{1-\mathrm{x}} \mathrm{Fe}_{\mathrm{x}}\right)_{2} \mathrm{Si}_{2}$ single crystals in fields applied along the $c$ axis at $1.5 \mathrm{~K}$. 


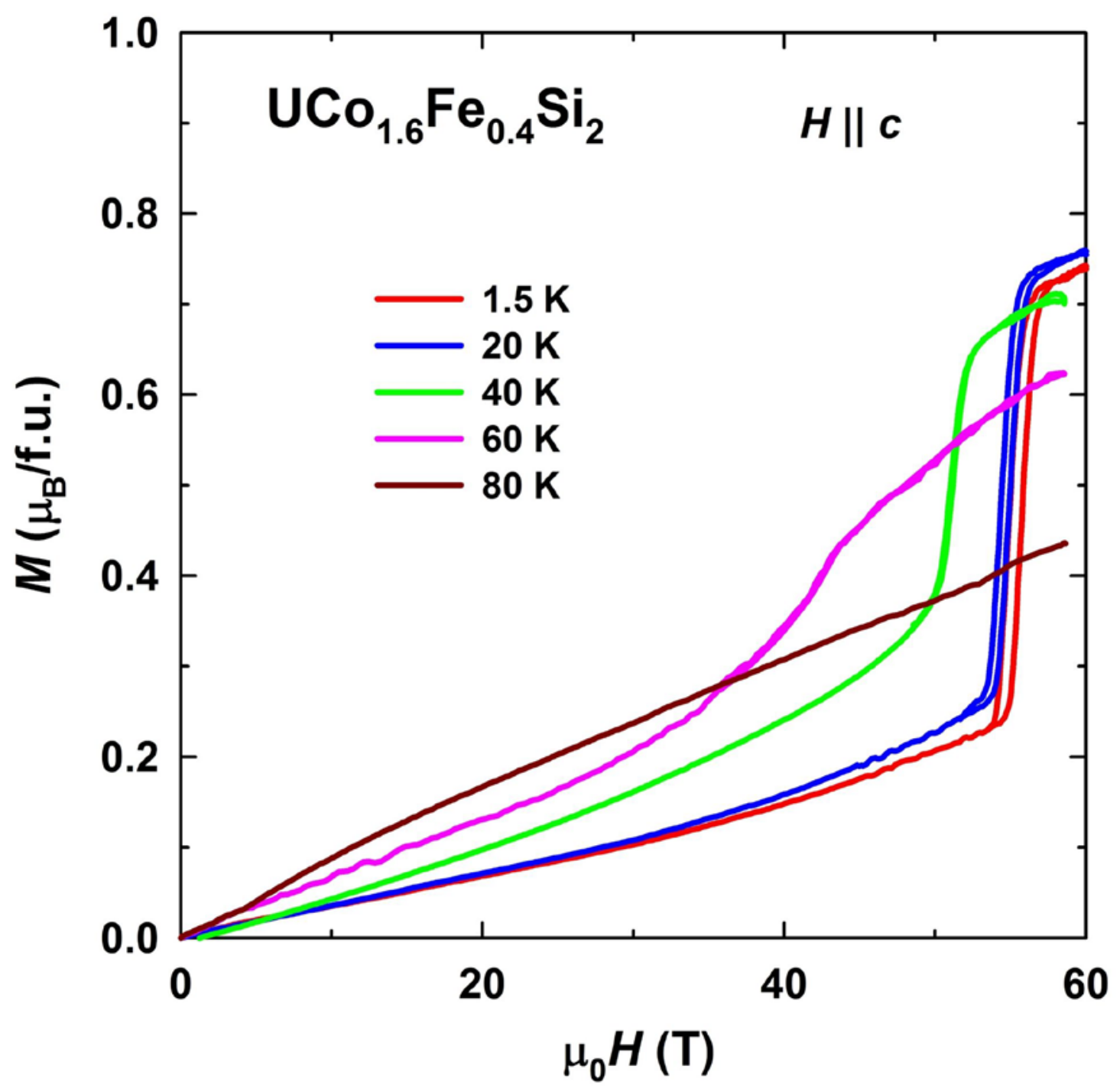

Fig. 7. Magnetization curves of a $\mathrm{UCo}_{1.6} \mathrm{Fe}_{0.4} \mathrm{Si}_{2}$ single crystal in fields applied along the $c$ axis at several temperatures. 


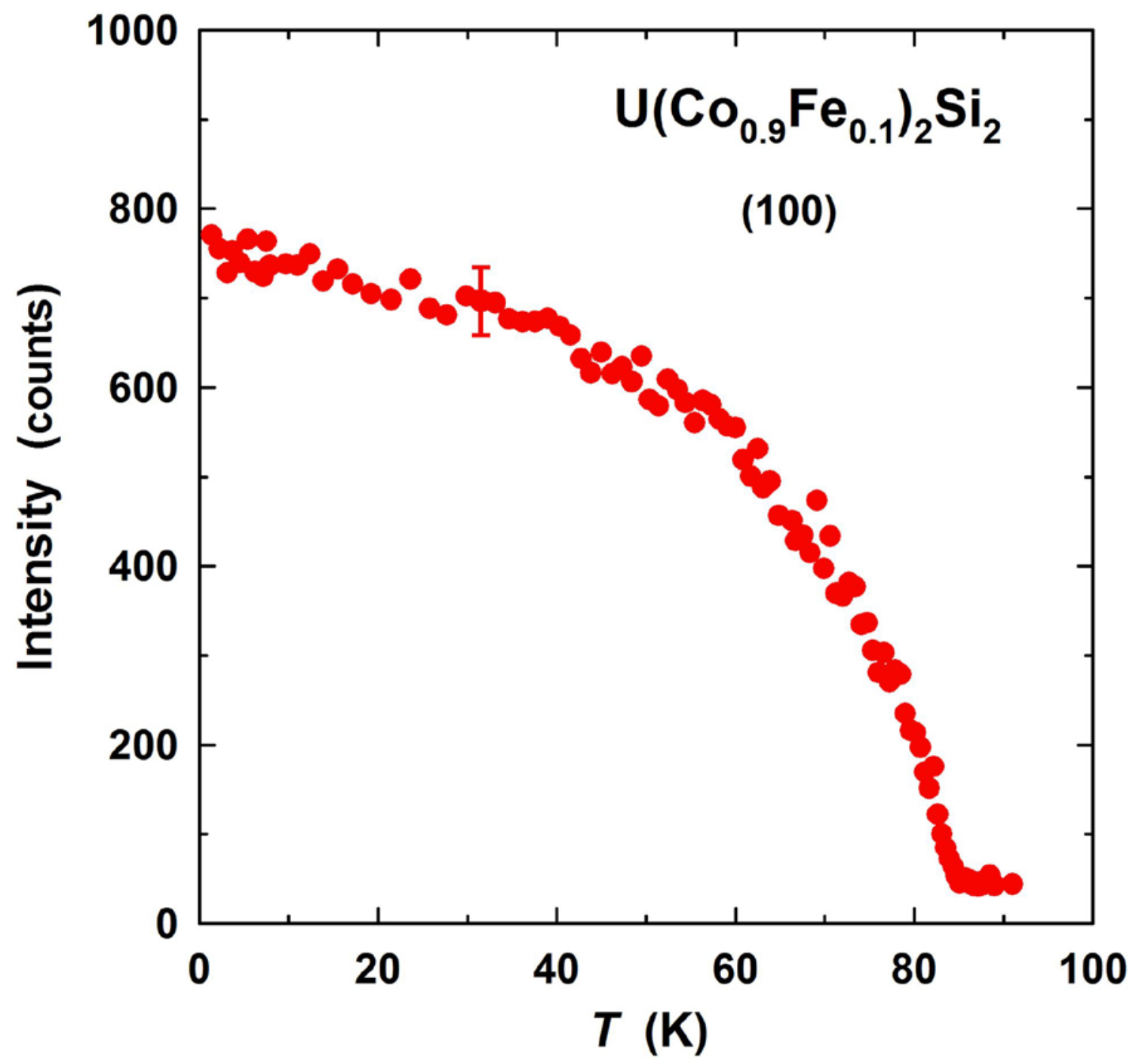

Fig. 8. The temperature dependence of the intensity of the (100) magnetic Bragg reflection in a $\mathrm{UCo}_{1.8} \mathrm{Fe}_{0.2} \mathrm{Si}_{2}$ single crystal. 